\title{
Erratum: Data Page: State of the biotech sector
}

\section{Stacy Lawrence}

Nat. Biotechnol. 22, 798 (2004)

The graph of "Number of biotech employees" at the bottom left contains two errors in labeling. The ' 0 ' entry against Asia should read "Not Available." The labels for the US and Europe should be reversed so that Europe has 11,449 and 32,470 employees and the US has 106,000 and 146,100 employees in 1998 and 2003, respectively.

\section{Erratum: Response to Stem cell differentiation}

Jeffrey Rothstein \& Evan Snyder

Nat Biotechnol. 22, 805-806 (2004)

The response to Salim et al.'s correspondence should not have included Jeffrey Rothstein's name as an author. 